Gynecol Obstet Invest 1997;43:283-284

\title{
Subject Index Vol. 43, 1997
}

Gynecologic and Obstetric Investigation

Aberrant umbilical vessel 232 Abnormal pregnancy 155 Abortion 131

- $\quad$ patients 84

Adenovirus 268

Adolescence 278

Adrenocortical steroids 41

Adults 206

Age 162 Aminetest 49 Amniotic fluid 29 Androstenedione 47 Anesthesia 41 Animal experiment 150 Antenatal diagnosis 232

- $\quad$ medical supervision 112

Anticancer chemotherapy 195

Antithrombin III 29

Asphyxia 94

Bacterial vaginosis 49 Bone mineralization 236 Bromocriptine 162

c-fos overexpression 200 C-reactive protein 225 CA125 125

Calcium homeostasis 236 Carbon dioxide 150

- tension 1 Carcinoma, endometrial 178 Carcinosarcoma 139 Cathepsin-D 125 Cervical cancer

200

human papillomavirus infections 49

mucus 166

Chorionic arteries and veins 219 Cisplatin-based chemotherapy 212 Clomiphene citrate

challenge test 186 Color Doppler 131 Concurrent chemoradiation 212 Condyloma, flat 255 Cord

blood 94, 155 Cystosarcoma phylloides 278

Danazol 6

Dehydroepiandrosterone sulfate 47 Dopaminergic neurotransmission 162 Doppler ultrasound 135

- velocimetry 55

Ectopic pregnancy 204 Emesis 195

Endogenous opioids 162 Endometrial histology 60 Endometriomas 53 Endometriosis 53,192,245

Endometrium 245 Endothelin 37 EPHgestosis 37

Epidermal growth factor receptor 125 Estrogens 41

Factor analysis 171 Fallopian tubes 116 Fetal 1

blood 158

sheep 150

weight estimation 98

- -, symphysis-fundus height 20

well-being 25 Fetoplacental circulation 11 Follicle-stimulating hormone 76, 242 Fura-2 145

Ganciclovir 268 Gene therapy 268 Germ cell tumors 68 Gestational age, fetal weight 20

Gestrinone 6, 192 Giant fibroadenoma 278 GnRH agonist 215 
analog 251

antagonist 215 Gonadotropins 242

-, bioactive and immunoactive 186 Granisetron 195 Gray-scale ultrasound 135 Gynecological surgery 41

Hematologic measurements 104

High estradiol 242

Histologic chorioamnionitis 225

HLA-DR 245

Human chorionic gonadotropin 76

decidua-associated protein 200178

embryo 73 Hypercoiled cord 64 Hyperplasia, endometrial 178 Hyperprolactinemia 183

Hysterosalpingogram 116 Hysterosalpingography 79

Ileus 206

Immunocytochemistry 125 Immunohistochemistry 89

Implantation 73

In vitro fertilization 242

- -, embryo transfer 142 Infertility 79, 171 Information 84 Innervation 89 Interferon- $\gamma 245$

Interleukin-1 166

receptor antagonist 166 Interleukin-4 155 Interleukin-6 225 Interleukin-10 155 Intracellular free calcium 145 Intraendometrial embryo transfer 73 Intraplacental Doppler waveforms 11

Intrauterine growth retardation 64

Labour 29 Leukocytes 104 Lipid peroxide 158 Literacy 112 Luteinizing hormone 162

Magnesium 120

- $\quad$ sulfate 236

Magnetic resonance imaging 135, 139 Malrotation 206 Maternal 1

- $\quad$ blood supply 89

Meckel-Grüber syndrome 142

Meta-analysis 79

Methadone-maintained pregnancies 25 Methotrexate 204 Methylprednisolone 195

Mode of delivery 104

Monocytes 6

Moos Menstrual Distress Questionnaire

score 120 Mother's nausea 108 Multiple follicles 242 Multivariate analysis 261 Myoma 55

Myomectomy 251 Myometrium 145

Naloxone 162

Nausea, previous pregnancy 108

Neonatal mortality 112

Nicaragua 112

NO donors 219

Nonsmoking 108

Nonstress tests 25

KAR, GEIV

E-Mail karger@karger.ch Fax+ 41613061234 http://www. karger. ch

(C) 1996 S. Karger AG, Basel

283 
Obstructed hemivagina 209 Oocyte donation 215 Oral contraceptives 276 Ovarian Brenner tumor of low malignant potential 135

cancer 268

carcinoma, risk factors 125

hyperstimulation 215 Ovary 68

Ovulation 166 Oxytocin 145

Pain 1 lóPenile intraepithelial neoplasia

255

Peoscopy 255

Perimenopause 186

Pharmacologic treatment, fibroids 251

Placenta 11

Placental disease 11

remnant 131

weight 108 Polycystic ovary 183

Poorly differentiated adenocarcinoma of

unknown origin 212 Postmenopausal endometrium 178

tamoxifen 60 Postmenopause 41 Preeclampsia 89, 158,219 Pregnancy 37,89,219

nausea 108

rate 79

Premature ovarian failure 47 Prematurity 98

Premenstrual distress 120

syndrome 120 Prenatal diagnosis 98 Preterm delivery 225

infant 236

labor 34 Progesterone 34 Prognostic value 79 Prolactin 183 Prostaglandin E2 145

F2 $\alpha 145$ Psychological test 171 Pulsed Doppler method 64 Pyridoxine 120

Recurrence rate 53 Renal agenesis 209 Reproductive years 162 Ritodrin 34

Sheep 1

Sigmoid volvulus 204

Signs 49

Size reduction, uterine leiomyoma 276

Solitary peritoneal metastasis 212

Soluble interleukin- 6 receptor 225

- $\quad$ tumor necrosis factor receptors 155

Sonohysterography 131

Sonohysterosalpingography 116

Squamous cell carcinoma, vulva 261

Stress 171

Strumaovarii 68

Symphysis-fundus height, fetal weight estimation 20 Symptoms 49

T cells 245 Testosterone 47

Thrombin-antithrombin III complexes 29 Thymidine kinase 268 Thyrotrophin 94

Transcutaneous Pco? 150

- $\quad$ tension 1

Twin pregnancy 142

Ultrasonography 98 Ultrasound 116,232 Umbilical cord occlusion 150 
venous pulsation 64 Unilateral breast enlargement 278 Univariate analysis 261 Unstimulated in vitro fertilization 76 Uterine artery 55

fluid 178

leiomyomata 251,276

volume 55 Uterus 139

didelphis 209

Vaginal breech delivery 94

wet smear 49

Verapamil 6

Yeast 120

284

Gynecol Obstet Invest Vol. 43, 1997

Subject Index 